PALAVRAS. Revista de Epistemología, Metodología y Ética del Psicoanálisis

ISSN: $2468-9831$

www.revistas.unlp.edu.ar/palavras

palavras@outlook.com.ar

Argentina

\title{
PSICOSIS Y EPISTEMOLOGÍA: LA POSICIÓN SUBJETIVA DEL PARANOICO Y SU RELACIÓN CON LA COSA (DAS DING)
}

\author{
DOI $10.24215 / 24689831 \mathrm{e} 003$ \\ Ignacio Iglesias Colillas
}

\begin{abstract}
Departing from a detailed study of Lacan's VII ${ }^{\circ}$ seminar, the present work proposes to base the following hypothesis: as well as Kant and his "third antinomy of pure reason" allows Lacan to base, from the epistemological conditions, das Ding as a concept, from clinic, is the subjective position in paranoia one of the most important arguments. The assumptions that pretend to fundament this interpretation will be unfolded in a series of successive and logically complementary movements.

In the first one we will follow the lacanian study of Freud's "Project of psychology" from which is located one of the first conceptual developments of das Ding.

In the second one we will mention the most relevant highlights of kantian's concept of "thingas-such" and we will elucidate how Lacan uses the third antinomy of pure reason with the purpose of showing the impasse in the interrogation of the cause, topic related directly with das Ding.

In the third one we intend to indicate and clarify the argumentative value of some of the references made by Lacan to Heidegger"s concept of "Thing". At last, in the fourth movement we will put in relief the most significant quotes about paranoia in this seminar so as to highlight the value of the subjective position in paranoia as a central issue to state das Ding as a concept.
\end{abstract}

Key words: das Ding - paranoia - epistemology ethics
Partiendo de un detallado estudio del seminario VII de Lacan, el presente trabajo se propone fundamentar la siguiente hipótesis: así como Kant y la tercera antinomia de la razón pura le permiten a Lacan fundamentar, desde las condiciones epistémicas, el concepto de das Ding, desde la clínica, es la "posición del sujeto en la paranoia" uno de sus argumentos principales. Los postulados que pretenden fundamentar esta lectura se desplegarán en una serie de movimientos sucesivos y lógicamente complementarios.

En el primero ubicaremos el abrevar lacaniano en el "Proyecto de Psicología" a partir del cual se ubica uno de los primeros desarrollos freudianos de das Ding.

En el segundo haremos mención de algunos puntos fundamentales del concepto kantiano de "cosa en sí" y exploraremos cómo utiliza Lacan la tercera antinomia de la razón pura a los fines de ubicar el impasse en la pregunta por la causa, temática relacionada con das Ding.

En el tercero pretendemos señalar y explicitar el valor argumentativo de algunas de las referencias hechas por Lacan al concepto de "Cosa" en Heidegger.

Por último, en el cuarto movimiento pondremos en relieve las diversas referencias que hace Lacan a la paranoia en este seminario para así poder destacar el valor que tiene la posición del sujeto en la paranoia en tanto postulado central en la fundamentación de das Ding como concepto.

Palabras clave: Das Ding - paranoia epistemología - ética

Cómo citar este artículo:

Iglesias Colillas, I. (2015). Psicosis y epistemología: la posición subjetiva del paranoico y su relación con la Cosa (das Ding). Palavras. Revista de Epistemología, Metodología y Ética del Psicoanálisis, 1(1), 60-82. Recuperado de www.revistas.unlp.edu.ar/palavras 


\section{PSICOSIS Y EPISTEMOLOGÍA: LA POSICIÓN SUBJETIVA DEL PARANOICO Y SU RELACIÓN CON LA COSA (DAS DING)}

Ignacio Iglesias Colillas *

\section{Introducción y posicionamiento de la problemática}

"Pues bien, el paso dado, a nivel del principio del placer, por Freud, es mostrarnos que no existe Soberano Bien-que el Soberano Bien, que es das Ding, que es la madre, que es el objeto del incesto, es un bien interdicto y que no existe otro bien. Tal es el fundamento, invertido en Freud, de la ley moral" (Lacan, 2003: 88).

El presente trabajo se propone fundamentar la siguiente hipótesis: así como Kant y la tercera antinomia de la razón pura le permiten a Lacan fundamentar -desde las "condiciones epistémicas" y sus paradojas- el concepto de Das Ding (Vassallo, 2009), desde la clínica, es la "posición del sujeto en la paranoia" uno de los argumentos centrales. En el seminario VII, dedicado precisamente a la ética del psicoanálisis, Lacan conceptualiza una modalidad de "primer emplazamiento de la orientación subjetiva" (Lacan, 2003: 70), entendiéndola como "una relación a ese Das Ding original" (Ibidem).

Nuestra hipótesis principal presupone apoyarnos en otra hipótesis auxiliar: Lacan utiliza una estrategia argumentativa mixta o de al menos dos niveles: el clínico y el epistemológico. Esta era una estrategia argumentativa bien conocida por Freud, utilizada por ejemplo en el capítulo I de Introducción del narcisismo al postular una "homología libidinal" entre la vida animica de los niños, de los pueblos primitivos y... los esquizofrénicos (Freud, 2000a: 73).

\footnotetext{
Facultad de Psicología de la Universidad de Buenos Aires, Argentina. Mail: ignaiglesias@yahoo.com.ar
} 
También la podemos ubicar -más nítidamente aún- en Más allá del principio del placer. Con miras de fundamentar el concepto de "pulsión de muerte", en el capítulo IV de dicho ensayo Freud pone en diálogo las tesis kantianas sobre el tiempo -en pos de introducir lo Zeitlos (atemporal) de lo inconsciente- (Freud, 2001c: 28) con la función de los sueños en las neurosis traumáticas, cimentando las bases que, al final del capítulo, plantearán estrechos vínculos entre la "compulsión de repetición" y la "pulsión de muerte" por un lado, y por el otro, la relación inversa entre esquizofrenia y enfermedad orgánica (Ibídem: 32). Esto implica que reunir a Kant con ciertos fenómenos de las psicosis no es algo que no pueda encontrarse ya en Freud, y Lacan no hace más que continuarlo en este sentido.

Este tipo de estrategia argumentativa, que incluso a veces pone en diálogo una doctrina epistemológica como la de Kant con ciertos fenómenos clínicos de las psicosis, ha sido exhaustivamente estudiada por P. Ricoeur al ocuparse sobre el problema epistemológico del freudismo. Ricoeur sostiene que "los escritos de Freud se presentan a la primera mirada como un discurso mixto, incluso ambiguo, que lo mismo enuncia conflictos de fuerza que serían de la competencia de una energética, como relaciones de sentido que corresponderian a la jurisdicción de una hermenéutica (...). Ese discurso mixto constituye la razón de ser del psicoanálisis" (Ricoeur, 2009: 60). Cabe destacar que Ricoeur nunca reduce el psicoanálisis a "una hermenéutica", y aunque ése hubiera sido el caso, resta aún una profunda discusión filosófica sobre cómo entendemos ése término. La "hermenéutica" a la cual hace referencia Ricoeur se encuentra mucho más próxima a la "fenomenología hermenéutica" de Heidegger que a la "hermenéutica romántica" de Dilthey y Schleirmacher. Ricoeur siempre sostuvo que el psicoanálisis se trata de un discurso mixto, un discurso que articula "fuerza" y "significación", en términos de Derrida (Derrida, 1989). Veremos a lo largo de este breve ensayo el rendimiento teórico de estos postulados. 
Esta hipótesis postula que si el estatuto epistemológico del psicoanálisis es "mixto", cabría esperar que las estrategias argumentativas presenten a su vez dicho carácter. Los argumentos que pretenden fundamentar esta lectura se desplegarán en una serie de movimientos sucesivos y lógicamente complementarios.

En el primero ubicaremos la lectura lacaniana del Proyecto de Psicología de Freud a partir del cual se ubica uno de los primeros desarrollos freudianos de Das Ding. Sostenemos que es la lectura lacaniana la que, resignificando el concepto freudiano de Das Ding, lo pone en relación con la posición subjetiva del paranoico.

En el segundo haremos mención de algunos puntos fundamentales del concepto kantiano de "cosa en sí y exploraremos cómo utiliza Lacan la tercera antinomia de la razón pura a los fines de ubicar el impasse en la pregunta por la causa, temática que también se entronca con la noción de Das Ding.

En el tercero pretendemos señalar y explicitar el valor argumentativo de algunas de las referencias a Heidegger hechas por Lacan en el seminario VII, especialmente las que pueden rastrearse hasta los cursos de 1935/6 reunidos bajo el título "La pregunta por la cosa" y la conferencia de Heidegger sobre "La cosa" de 1950.

Por último, en el cuarto movimiento pondremos en relieve las diversas referencias que hace Lacan a la paranoia en el seminario VII para así poder destacar el valor que tiene la posición del sujeto en la paranoia como postulado central en la fundamentación de Das Ding como concepto.

Antes de avanzar en el desarrollo de los cuatro movimientos propuestos más arriba, cabe aquí introducir y comentar cuáles son las tesis principales de Lacan en este seminario sobre la ética del psicoanálisis.

Leemos así en la página 30 de dicho seminario: "mi tesis es que la ley moral, el mandamiento moral, es aquello por lo cual, en nuestra actividad 
en tanto que estructurada por lo simbólico, se presentifica lo real -lo real como tal, el peso de lo real. Tesis que puede parecer a la vez una verdad trivial y una paradoja. Mi tesis implica, en efecto, que la ley moral se afirma contra el placer (...). Pues lo que puede constituir el alcance de esta mira reside justamente en el sentido que se dé, en el orden de las categorias que les enseño, en función de nuestra praxis de analistas, a ese término de real" (Lacan, 2003: 30-31. Cursivas añadidas).

Unas cuantas páginas antes Lacan propone: “la cuestión ética (...) se articula a partir de una orientación de la ubicación del hombre en relación con lo real. Para concebirla hay que ver qué sucedió en el intervalo entre Aristóteles y Freud" (Ibidem: 21).

¿Qué significa en este contexto la frase "en función de nuestra praxis de analistas" y que "la cuestión ética (...) se articula a partir de una orientación de la ubicación del hombre en relación con lo real"? Transformar estas dos frases en interrogantes puede servirnos a los fines de desplegar nuestro recorrido.

Lacan sostiene que "la cuestión ética, en la medida en que la posición de Freud nos permite progresar en ella, se articula a partir de una orientación de la ubicación del hombre en relación con lo real" (Lacan, 2003: 21. Cursivas añadidas). Es en este sentido que Lacan sugiere e invita a explorar "qué sucedió en el intervalo entre Aristóteles y Freud" (Ibídem), así como también planteó que "los límites éticos del análisis coinciden con los límites éticos de su praxis. Su praxis no es más que un preludio a una acción moral como tal -siendo la susodicha acción aquella por la que desembocamos en lo real" (Ibidem: 32).

\section{Das Ding y el "Complejo del prójimo"}

Haciendo una minuciosa lectura del "Proyecto de psicología" de Freud, Lacan introduce -en el capítulo IV, “Das Ding (I)"- la Cosa freudiana. Dice 
Lacan: "si introduzco este término, es porque hay ciertas ambigüedades, ciertas insuficiencias en lo concerniente al verdadero sentido en Freud de la oposición entre principio de realidad y principio del placer -es decir, de aquello en cuya pista intento introducirlos este año, para hacerles comprender su importancia para nuestra práctica en tanto que ética, y cómo esas ambigüedades se deben a algo que es del orden del significante e incluso del orden lingüístico" (Ibídem: 57. Cursivas añadidas).

Si bien Lacan comienza diciendo que "el Entwurf es la teoria de un aparato neuronal, respecto al cual el organismo permanece exterior, al igual que el mundo externo" (Ibídem: 61), inmediatamente después -luego de comentar la importancia de las diversas Niederschrift ${ }^{1}$ y la interposición de la estructura significante entre la percepción y la consciencia (Ibidem: 66)- se ocupa de desplegar y desarrollar el concepto del Nebenmensch o Complejo del prójimo (Ibídem: 67).

Según Lacan se trata de una fórmula asombrosa que "articula poderosamente lo marginal y lo similar, la separación y la identidad" (Ibídem), y también es lo que permite la "primera aprehensión de la realidad por el sujeto" (Ibídem). Ahora bien, ¿qué significa esto?

"El Ding -continúa Lacan- es el elemento que es aislado en el origen por el sujeto, en su experiencia del Nebenmensch, como siendo por naturaleza extranjero, Fremde" (Ibídem). Consideramos que la lectura de Lacan apunta a mostrar cómo en el Proyecto de psicología Freud sostiene, a contrapelo de muchas lecturas que se hacen de este ensayo (reducirlo a un texto subsumible en el marco de las Ciencias Naturales, a un "aparato neuronal", etc.), que todos los conceptos esgrimidos aquí por Freud se extienden y desarrollan dentro de otro concepto más amplio, de carácter "relacional", que es el "Complejo del prójimo". El Gran Otro (A) es constituyente del sujeto. Das Ding es el Otro Absoluto del sujeto, y lo constituye siendo exterior.

1 "Niederschrift": "Precipitado de escritura", literalmente. "Transcripción". 
E1 "desamparo", la "acción específica", las "vivencias de dolor" y de "satisfacción" así como la "inervación lingüística" -que queda incluida en la "acción específica" (Freud, 2001b: 414) son considerados "dentro" o "referidos a" el "Complejo del prójimo" (Ibídem, 376 y ss.)².

Dicho Complejo del prójimo no es un "accesorio" en un aparato neuronal que flota en las tinieblas originarias, sino que es el lugar mismo donde se da la constitución subjetiva, la fundación de lo psíquico en tanto tal ${ }^{3}$. El "psiquismo" en Freud nunca está "adentro" de un "individuo" en sentido biológico. Lo "psíquico" se da en el "entre». Esta es, según nuestra opinión, una de las razones fundamentales que llevaron a Lacan hacia la topologia: "el inconsciente está estructurado como un lenguaje": ni adentro ni afuera, "entre".

Es más, es aquél Complejo del prójimo el que "se separa en dos componentes, uno de los cuales impone por una ensambladura constante, se mantiene reunido como una cosa del mundo, mientras que el otro es comprendido por un trabajo mnémico, es decir, puede ser reconducido a una noticia del cuerpo propio" (Freud, 2001b: 377). Es a partir de leer esta separación en dos del Complejo del prójimo que Lacan construye en el Seminario VII -apelando también a otras referencias - el concepto de Das Ding.

Este recorrido lo lleva a Lacan a sostener que hay una división original de la experiencia de la realidad (Lacan, 2003: 67). "El Ding es el elemento que es aislado en el origen por el sujeto, en su experiencia del Nebenmensch, como siendo por naturaleza extranjero, Fremde. El complejo del objeto está en dos partes, hay división, diferencia en el enfoque del juicio. Todo lo que en el objeto es cualidad, puede ser formulado como

\footnotetext{
2 Tal es así que Lacan sostuvo que de lo que se trata en el Entwurf, de lo que palpita en su fondo, es "una experiencia de orden moral" (Lacan, 2003: 41). O bien: "la experiencia de satisfacción del sujeto está enteramente suspendida del otro" (Ibidem: 53).

3 El Complejo del prójimo podría relacionarse -epistemológicamente- con el "Ser - con" en tanto elemento existenciario en la analítica del Dasein en "El ser y el tiempo" de Heidegger (Heidegger, 2009: 142).
} 
atributo, cae dentro del sistema $\psi$ y constituye las Vorstellungen primitivas alrededor de las cuales se jugará el destino de lo que está reglado según las leyes del Lust y del Unlust, del placer y del displacer, en lo que se puede llamar las informaciones primitivas del sujeto. Das Ding es algo totalmente diferente. Esta es una división original de la experiencia de la realidad" (Ibídem). Lacan no deja de agregar que esta división original es vuelta a encontrar también en la Verneinung, agregando que se trata de "la misma función de aquello que, desde el interior del sujeto, resulta llevado en el origen a un primer exterior" (Ibídem). Retomaremos este punto más adelante al abordar la cuestión de la paranoia.

\section{La cosa en si y las antinomias kantianas de la razón pura}

Como lo ha demostrado cabalmente la Dra. Vassallo en su artículo "El impasse conceptual de la causa en Lacan y las antinomias kantianas", Lacan es deudor de Heidegger; no solamente en cuanto a su posicionamiento y uso de las filosofias, sino especial y particularmente en la lectura que hace de Kant. Entre otras cosas, esto se explica "porque la filosofia trascendental de Kant reúne todas las condiciones para refutar el empirismo chato" (Vassallo, 2009: 2). Y también es crucial el recurso a Kant a la hora de fundamentar el concepto de Das Ding.

Es esa "realidad muda que es Das Ding -a saber la realidad que comanda, que ordena. Esto es lo que asoma en la filosofia de alguien que, mejor que nadie, entrevió la función de Das Ding, aunque sólo la abordó por las vías de la filosofia de la ciencia, a saber, Kant. Es a fin de cuentas concebible que sea como trama significante pura, como máxima universal, como la cosa más despojada de relaciones con el individuo, como deben presentarse los términos de das Ding" (Lacan, 2003: 71). ¿La "máxima universal" como "trama significante pura"? 
Unas líneas más adelante Lacan aclara que "la Cosa sólo se nos presenta en la medida en que hace palabra (...). En el texto de Freud, la manera en que lo extranjero, lo hostil, aparece en la primera experiencia de la realidad para el sujeto humano, es el grito" (Ibídem).

Pero detengámonos un poco en las antinomias de la razón pura. El núcleo central de estos problemas es expuesto por Vassallo en el artículo mencionado cuando analiza en detalle las consecuencias de la tercera antinomia en el pensamiento de Lacan. Lo fundamental es precisamente la articulación entre Kant y Lacan -vía Heidegger- en cuanto al pensamiento de la causalidad, especialmente en cuanto a sus impasses.

Retomaremos entonces algunos de los argumentos centrales de dicho ensayo. "En el seminario De la esencia de la libertad humana (1930), donde Heidegger desmenuza la tercera de las cuatro antinomias, resume ese conflicto interno a la razón diciendo: "eso mismo que ignoramos, no dejamos, sin embargo, de concebirlo". Se abre, en consecuencia, una brecha entre el conocer o entendimiento (Verstand) y la Razón (Vernunft) (...). Denominada también antinomia de la naturaleza/libertad, Kant la desarrolla en dos partes (tesis y antítesis, seguidas ambas de una prueba) ${ }^{4}$. La finalidad reside en resolver si existe una causa independiente del conjunto de los fenómenos del mundo (tesis) o si estos, lejos de estar regidos por una causa que les es exterior, solo obedecen a una cadena de causas-efectos intramundanos infinita, de tipo natural (antítesis)" (Vassallo, 2009: 3).

$\mathrm{Si}$ hubiera solamente causas naturales, deberíamos admitir un encadenamiento interminable de causas y efectos. "Para salir de esa cadena sin fin, interna al mundo, se hace necesario concebir una causa que salga de la "serie" de los fenómenos. Esa causa libre debería contener una "espontaneidad" que haga de "comienzo absoluto" de la serie

\footnotetext{
4 Véase Kant, “Crítica de la razón pura”, págs. 526-531 (Kant, 2004: 526-531).
} 
intramundana" (Ibídem). Kant concluye que es necesario suponer a priori una causalidad libre denominada "libertad trascendental".

Según H. Allison, un acérrimo "defensor" de Kant, en la "Dialéctica" de la "Crítica de la razón pura" la libertad trascendental "es definida como espontaneidad absoluta, y esto se entiende en términos esencialmente negativos, como un poder causal que en sí mismo es independiente de la determinación de causas antecedentes" (Allison, 1992: 479). Pero Allison advierte unas páginas más adelante que esta no es la única interpretación posible: "también es posible considerar que Kant está afirmando una conexión necesaria entre el concepto de libertad práctica y la idea trascendental y no entre la realidad de los dos tipos de libertad. En efecto, esto es precisamente lo que Kant dice cuando señala que "el concepto de libertad práctica se basa sobre esta idea trascendentalı. Si tomamos esto seriamente -sigue Allison- lo que Kant sostiene es que la concebibilidad de la libertad práctica implica una referencia a la idea trascendental. En términos kantianos, la idea de libertad trascendental tiene una función regulativa respecto de la concepción de la libertad práctica. Esto concuerda con la función de modelo asignada a la idea en la observación de la tesis de la "Tercera antinomia". También es compatible con el "Canon", pues deja abierta la posibilidad de que seamos libres en sentido práctico pero no en sentido trascendental" (Ibidem: 483).

Lo interesante es cómo -siguiendo a Vassallo nuevamente-, aunque se ignore qué es la causa en sí misma, "se hace evidente la necesidad de postularla (...). En la "Crítica de la razón práctica", Kant la sitúa en una voluntad humana autónoma y no subordinada a la ley causal de tipo natural que regiría los "fenómenos" (...). En el campo de la razón práctica, o sea, de la moral, la serie de los fenómenos llevan un nombre: lo "patológico" (Vassallo, 2009: 4). Es decir, "pertenecemos al registro de lo fenoménico en tanto nuestras conductas participan de la serie intramundana de las causas naturales o stimuli, es decir, al registro de lo patológico. Pero pertenecemos también al registro de lo noumenal o extra- 
mundano, que no está sometido al principio de causalidad natural verificable en la experiencia. Ese no sometimiento a lo patológico se manifiesta en la ley moral, autónoma y puramente formal respecto de lo patológico (es precisamente esa autonomía la objetada por Freud en " $E l$ yo y el Ello"). Según Kant, escuchamos la "voz" del imperativo categórico (...) con independencia de toda inclinación de lo sensible” (Ibidem).

Lo que Lacan extrae de este recorrido es que esta división entre la ley moral universal y "lo patológico" en sentido kantiano, exhibe una hiancia, sintomática, donde aparece precisamente la división del Sujeto ante el “objeto $a$ ". Lacan se apoyaría entonces también en esta antinomia para cimentar lo que posteriormente será su “objeto a", uno de los desarrollos posteriores de lo que en el seminario VII es conceptualizado como Das Ding.

Los siguientes parágrafos del Seminario X ilustran bien este punto:

"El a es la causa, la causa del deseo. Les indiqué que no es una mala forma de comprenderlo volver al enigma que nos plantea el funcionamiento de la categoría de la causa, porque está muy claro que, cualquiera que sea la critica, el esfuerzo de reducción, fenomenológico o no, que le apliquemos, esta categoría funciona, y no como una etapa únicamente arcaica de nuestro desarrollo. Esta categoría, pretendo transferirla del dominio que llamaré, con Kant, la estética trascendental a lo que llamaré, si tienen ustedes la bondad de consentirlo, mi ética trascendental.

“(...) que los filósofos hagan su trabajo y osen formular algo que permita situar verdaderamente en su lugar la operación que les indico a ustedes cuando digo que extraigo la función de la causa del campo de la estética trascendental, la de Kant. Convendría que otros les indicaran que esto no es más que una extracción enteramente pedagógica, porque hay muchas otras cosas que conviene extraer todavia de esta estética trascendental” (Lacan, 2006: 304). 


\section{Heidegger y la "Cosa"}

Ya que trabajar y explicitar las relaciones entre Heidegger y Lacan podria requerir varias tesis doctorales, simplemente vamos a comentar sucintamente algunos puntos de interés a los fines de fundamentar las propuestas realizadas en este ensayo.

Como lo señalara Vassallo en el texto comentado más arriba, no es que Lacan copie a Heidegger, sino que toma -a nuestro criterio- el método de lectura y el tipo de cuestionamiento -o modalidad de la reflexión- que se muestra claramente de inspiración heideggeriana. Lo que se cuestiona primordialmente es que haya una relación directa "sujeto/objeto". Heidegger ha desarrollado estos interrogantes no solamente en el campo epistémico sino que ha reconducido estas preguntas al ámbito de la analitica del Dasein.

Detengámonos en algunas de las reflexiones de Heidegger en "La pregunta por la cosa". Dice el autor en el punto 2, "Las múltiples maneras de hablar de la cosa": "comprendemos la palabra "cosa" en dos significados, uno más restringido y otro más amplio. Cosa en el sentido más restringido es lo tangible, visible, etc., lo presente fáctico (Vorhandene). Cosa en sentido más amplio es todo asunto dispuesto de una u otra manera, las cosas que ocurren en el "Mundo", acontecimientos, sucesos. Por último, hay todavía un uso de la palabra en el sentido más amplio posible. Este sentido se prefiguró hace ya mucho y se hizo usual sobre todo en la filosofia del siglo XVIII. De acuerdo a él, habla por ejemplo Kant de "cosa en sî", distinguiéndola de "cosa para nosotros", es decir, de la cosa como "fenómeno". Una cosa en sí es aquella que no nos es accesible por la experiencia como lo son las piedras, las plantas y los animales (...). Cuando Kant dice que Dios es una cosa (...), Dios es una cosa en tanto es en general algo, una X" (Heidegger, 1975: 14-15). 
Heidegger llega así a distinguir tres significados de "cosa": "1. Cosa en el sentido de lo objetivamente presente (das Vorhandenen): piedra, trozo de madera, tenaza, reloj (...), etc. 2. Cosa en el sentido de lo antedicho pero además, planes, decisiones, reflexiones, mentalidad, hechos, lo histórico... 3. Todo esto, y además todo lo otro que es un algo cualquiera y no nada. Los límites en los que fijamos el significado de la palabra cosa, son siempre arbitrarios. Conforme a ellos cambia el ámbito y la dirección de nuestro pensar" (Ibídem).

Más adelante, en el punto 11 de estas lecciones, Heidegger postula que el concepto de "cosa" es indisociable de cierta noción de "proposición" y de cierta noción de "verdad". Examinemos este punto en detalle. Heidegger afirma que fue en la época de Platón y de Aristóteles en la que se formó "la determinación de la cosa como soporte de propiedades. En la misma época se llegó al descubrimiento de la esencia de la proposición. Contemporánea a ellas nació la caracterización de la verdad como adecuación del percibir a las cosas, verdad que tiene su sitio en la proposición (...). También podemos mostrar cómo éstas doctrinas sobre la cosa, la proposición, y la verdad, cambiaron en la Stoa, cómo en la escolástica aparecen de nuevo diferenciaciones, y otras aún en la época moderna, y luego en el idealismo alemán" (Ibidem: 45).

Pero a Heidegger no le interesa hacer una "historia" de la pregunta por la cosa, sino que apunta más bien a la siguiente pregunta que es al mismo tiempo una decisión: “¿es por casualidad que son contemporáneas la determinación de la esencia de la cosa y la determinación de la esencia de la proposición y la determinación de la esencia de la verdad, o se interrelacionan todas ellas necesariamente? $\mathrm{Si}$ así ocurre, ¿cómo se interrelacionan estas determinaciones?” (Ibídem). Da la impresión que la investigación heideggeriana apunta más a las condiciones de posibilidad de la pregunta -al cómo- que al qué del preguntar "qué es una cosa". Y prosigue unas líneas más adelante: "Hemos visto que la determinación de la estructura esencial de la verdad tiene que orientarse -sobre la base de la 
esencia de la verdad como certeza- en la estructura esencial de las cosas. Con ello se ha concretado una determinada conexión entre la esencia de la cosa y la esencia de la proposición y la esencia de la verdad” (Ibídem).

Este itinerario desemboca finalmente en la siguiente pregunta: “¿Extrajo el hombre la estructura de la proposición de la estructura de la cosa, o introdujo la estructura de la proposición en las cosas?" (Ibidem: 46). Como se notará inmediatamente, este tipo de preguntas hacen vacilar nuestra noción de "realidad". Y más aún: ¿estamos a la altura de poder definir "realidad" cuando se trata de un psicoanálisis?

Cabe preguntarse si el modo en que Lacan se interroga por el das Ding de Freud no es claramente de inspiración kantiana y heideggeriana. Esto se ve un poco más claramente en la conferencia de 1950 titulada "La cosa". Cuando Heidegger describe el método del "paso hacia atrás" (Heidegger, 2001: 133), ¿acaso no está preguntándose por cómo salirse del campo de la representación para acceder reflexivamente a "algo" que nos condiciona y nos determina -en el sentido estricto de la palabra “determinar"- pero que no es representable en sí mismo? ¿Acaso no es ése ámbito de lo irrepresentable el referente del concepto "objeto a"? Atengámonos por ahora a seguir pensando la Cosa (das Ding), siguiendo el despliegue de los argumentos del seminario VII en torno a las "representaciones - palabra" y las "representaciones - cosa", ya que en estos dos términos se continúa en Freud el problema kantiano sobre lo "representable".

Veamos cómo plantea Lacan la diferencia entre las Sachvorstellungen (representación-cosa) y las Wortvorstellungen (representación-palabra). Lacan se pregunta a qué denominamos "Sachvorstellungen", y dice: "nos vemos pues llevados a distinguir la articulación efectiva de un discurso, de una gravitación de las Vorstellungen bajo la forma de Vortellungrepräsentanzen de esas articulaciones inconscientes. Se trata de ver, en tales circunstancias, a qué denominamos Sachvorstellungen. Estas deben situarse en oposición polar con el juego de palabras, con las 
Wortvorstellungen, pero a este nivel, unas no son sin las otras. Das Ding es otra cosa -es una función primordial, que se sitúa en el nivel inicial de instauración de la gravitación de las Vorstellungen inconscientes (...). No se asombrarán -continúa Lacan- que les diga que, a nivel de las Vorstellungen, la Cosa no sólo no es nada, sino literalmente no está -ella se distingue como ausente, como extranjera. Todo lo que se articula de ella como bueno y malo divide respecto a ella al sujeto irrepresiblemente, irremediablemente diré, y sin ninguna duda en relación a la misma Cosa. No existen el objeto bueno y el objeto malo, existe lo bueno y lo malo y después existe la Cosa. Lo bueno y lo malo ya entran en el orden de la Vorstellung, están allí como índices de lo que orienta la posición del sujeto, según el principio del placer, en relación a lo que nunca será más que representación, búsqueda de un estado elegido, de un estado de anhelo, de espera, ¿de qué? De algo que siempre está a cierta distancia de la Cosa, aunque esté reglado por esa Cosa, la cual está más allá" (Lacan, 2003: 80).

Lacan sostiene explícitamente -siguiendo a Benveniste- que los procesos de pensamiento se reflejan en un discurso (Ibidem). Si no fuera así, ¿qué sentido tendría hablar de "indices" de lo que orienta la posición del sujeto"? Pero surge inmediatamente la siguiente pregunta: ¿acaso algo en la experiencia del análisis permite entrever el singular efecto que la Cosa puede ejercer en la economía misma de las palabras ${ }^{6}$ ? Este itinerario nos arroja directamente en el umbral del cuarto movimiento de nuestro ensayo, que es al mismo tiempo su centro de gravedad: esa experiencia a

\footnotetext{
5 ¿"Índice" no nos remite acaso a la extensa nota al pie que encontramos en "De una cuestión preliminar a todo tratamiento posible de la psicosis"? Dice Lacan en relación a la función de los "shifters": "Roman Jakobson toma este término de Jespersen para designar esas palabras del código que solo toman sentido por las coordenadas (atribución, fechado, lugar de emisión) del mensaje. Referidas a la clasificación de Pierce, son símbolos - indices. Los pronombres personales son su ejemplo eminente: sus dificultades de adquisición como sus déficit funcionales ilustran la problemática engendrada por esos significantes en el sujeto" (Lacan, 1987: 517).

6 Leemos en el seminario 3, "Las psicosis", en relación a la rúbrica del delirio y al discurso psicótico: "ese lenguaje, que puede engañarnos en un primer abordaje del sujeto, incluso a veces hasta en el más delirante, nos lleva a superar esa noción y a formular el término de discurso (...). La economía del discurso, la relación de significación a significación, la relación de su discurso con el
} 
la cual venimos haciendo referencia desde la filosofia de Kant y de Heidegger, es homóloga de la experiencia de la irrupción de la Cosa que podemos ubicar y localizar nitidamente en el análisis del discurso psicótico.

\section{La posición subjetiva del paranoico y el primer emplazamiento subjetivo}

Retomemos aquí la hipótesis central de nuestro ensayo. Quisiéramos poner en relieve que es a partir de reflexionar sobre la "psicología del psicótico" que Lacan se interroga por su tesis fundamental sobre la "sincronía primitiva del sistema significante" (Lacan, 2003: 82-83), así como también es la posición subjetiva del paranoico lo que le permite a Lacan fundamentar el concepto de Das Ding y cómo se distingue este término del sistema significante en tanto "realidad muda" que lo condiciona.

"Veremos desarrollarse toda la psicología del psicótico -dice Lacan- en la medida en que un término, que sostiene la base del sistema de las palabras a cierta distancia o dimensión relacional, puede ser rechazado algo falta, hacia lo cual tiende desesperadamente su verdadero esfuerzo de suplencia, de significantización. Los dejo con la esperanza de que quizá tendremos que volver a esto, y también a la explicación notable que hizo Laplanche del caso de una experiencia poética que lo despliega, lo revela, lo vuelve perceptible de un modo especialmente esclarecedor, a saber, el caso de Hölderlin. La función de ese lugar es ser lo que contiene las palabras, en el sentido en que contener quiere decir retener, gracias a la cual una distancia y una articulación primitivas son posibles, gracias a la cual se introduce la sincronía, sobre la cual puede luego desplegarse la 
dialéctica esencial, aquella en la que el Otro puede ser el Otro del Otro"7 (Ibidem).

La referencia al libro de Laplanche titulado "Hölderlin y el problema del padre” en el capítulo "Das Ding (II)” del Seminario VII no es incidental sino principal a la hora de fundamentar este concepto. En dicho libro Laplanche reflexiona sobre el concepto de forclusión y sus relaciones con el Nombre del Padre. Lo que Lacan comenta en su Seminario VII es la siguiente cita de aquél libro:

"mencionaremos aqui una de las dificultades que se presentan cuando se intenta profundizar la "preclusión": si se trata de la ausencia radical de un significante, por ejemplo del nombre de Padre, apenas sería posible encontrar un ejemplo en la psicopatologia; por el contrario, hablar del nombre del Padre y de su ausencia, es quizá en mayor medida indicar una cierta falla en el lenguaje que un agujero en el "puro" significante. Si el lenguaje es desde un primer momento intencionalidad, si solo a partir de esa intencionalidad es posible distinguir un significante $y$ un significado provistos de una cierta autonomía, la consideración de un significante "como tal" representaría quizás sólo un momento extremo de la abstracción, al que la psicosis se aproxima sin nunca alcanzarlo. Lo que falta cuando Schreber pronuncia la palabra Padre, y sin duda la pronuncia, debe aún ser determinado" (Laplanche, 1975: 59).

¿Cómo llega Lacan a este punto en su argumentación? En las primeras páginas del punto 2 de la clase que estamos comentando -Das Ding (II)-, el autor hace un distingo crucial entre "negación» (Verneinung) y "represión" (Verdrängung), postulando -siguiendo al pie de la letra la "Carta 52" de

\footnotetext{
7 Notemos que es exactamente en estos términos que Lacan define al Nombre - del - padre o Padre Simbólico en el Seminario V: "llamamos ley a lo que se articula propiamente en el nivel del significante, a saber, el texto de la ley. No es lo mismo decir que ha de haber ahí una persona para sostener la autenticidad de la palabra, que decir que algo autoriza el texto de la ley. En efecto, a lo que autoriza el texto de la ley le basta con estar, por su parte, en el nivel del significante. Es lo que yo llamo el Nombre del Padre, es decir, el padre simbólico. Es un término que subsiste en el nivel del significante, que en el Otro, en cuanto sede de la ley, representa al Otro. Es el significante que apoya a la ley, que promulga la ley. Es el Otro en el Otro" (Lacan, 2001: 150).
} 
Freud a Fliess- que la Verdrängung recae sobre las Vorstellungrepräsentanzen, mientras que la Verneinung opera en el nivel del discurso, es decir, sobre las Wortvorstellung. La Verneinung es caracterizada por Lacan como lo que está a la vez presentificado y renegado entre lo Preconsciente (Vorbewusstsein) y la Consciencia (Bewusstsein). El nudo del problema aparece cuando se pretende distinguir conceptualmente Das Ding de las Vorstellungrepräsentanzen, ya que en la lectura que Lacan hace del "Proyecto de psicología" de Freud, se homologan los "signos de percepción" (Wahrnehemungszeichen) (Lacan, 2003: 80) con "el sistema primero de los significantes", como veíamos más arriba al comienzo de este apartado.

Lacan entiende que lo que Freud llama "Vorstellungrepräsentanzen" responde a las leyes del significante: la representación es "algo que tiene la misma estructura -es éste el punto en que insisto- que el significante. Esto no es simplemente Vorstellung, sino, como Freud lo escribe más tarde, en su artículo Lo inconsciente, Vorstellungrepräsentanz, lo que hace de la Vorstellung un elemento asociativo, combinatorio" (Lacan, 2003: 78).

Es decir, sin estas distinciones Lacan no tendría dónde apoyar su diferenciación entre "Das Ding" y las "Vorstellungrepräsentanzen". Habiendo explicitado el contexto en el cual se despliegan estos argumentos, a continuación retomaremos en forma extensa cómo introduce Lacan su comentario sobre el libro de Laplanche. La cita es la siguiente:

"Ven representar en ese juego de la oca a la Verneinung, desde cierto punto de vista, la forma invertida de la Verdrängung y la diferencia de organización que hay entre ambas (...). Indico, para quienes esto resulta aún problemático, que tienen igualmente una correspondencia entre lo que se articula plenamente en el 
inconsciente, la Verurteilung ${ }^{8}$ y lo que sucede en el nivel que Freud distingue en la carta 52, en la primera significación significante de la Verneinung, la de la Verwerfung 9 .

Uno de ustedes, Laplanche (...), en su tesis sobre Hölderlin a la que espero nos dediquemos un día aqui, se interroga y me interroga acerca de qué puede ser esa Verwerfung diciendo -¿iSe trata del Nombre-de-Padre, como en la paranoia o del Nombre-del-Padre? Si se trata de esto, pocos ejemplos patológicos nos ponen en presencia de su ausencia, de su rechazo efectivo. Si es el Nombre-del-Padre, ¿no entramos en una serie de dificultades en lo concerniente al hecho de que siempre hay algo significado para el sujeto, que está ligado con la experiencia, y esté ésta presente o ausente, de ese algo que con algún título, en algún grado, llegó a ocupar ese lugar para él?

Ciertamente, esta noción de la sustancia significante no puede dejar de producir problemas para toda buena cabeza. Pero no olviden que tenemos que vérnosla con el sistema de las Wahrnehemungszeichen, de los signos de percepción, es decir con el sistema primero de los significantes, con la sincronía primitiva del sistema significante. Todo comienza en la medida en que al mismo tiempo, en la Gleichzeitigkeit ${ }^{10}$, pueden presentársele al sujeto varios significantes. A ese nivel el Fort es correlativo del Da. El Fort sólo puede expresarse en la alternancia a partir de una sincronía fundamental. A partir de esta sincronía algo se organiza, algo que el simple juego del Fort y del Da no bastaría para constituir" (Lacan, 2003: 82).

\footnotetext{
8 Verurteilung: "juicio de condenación", también llamado por Freud "Urteilsverwerfung". Véase "La negación" (Freud, 2000b).

9 Aquí Lacan está diciendo explícitamente que la Verwerfung es una modalidad de la Verneinung. La llamada "forclusión" del Nombre del Padre es una modalidad de negación, tema que dejaremos de lado en el presente estudio.

10 Gleichzeitigkeit: articulación según una asociación por simultaneidad, referida en Freud a la primera transcripción de las percepciones, por completo insusceptibles de consciencia (Freud, 2001a: 275).
} 
Por último, queda por precisar cómo el sintagma "posición del sujeto" es utilizado por Lacan en relación a una "realidad ética", especialmente ubicada en la posición propia del paranoico:

"Para ir rápido -tan rápido como Freud en sus primeras percepciones de la realidad ética, en tanto ésta funciona en el sujeto con el que se enfrenta-evocaré la posición del sujeto en la tercera de las grandes categorias que Freud discierne primero -histeria, neurosis obsesiva y paranoia-. En la paranoia, cosa curiosa, Freud nos aporta este término, que les ruego mediten en su surgimiento primordial, Versagen des Glaubens. En ese primer extraño, respecto al cual el sujeto debe ubicarse de entrada, el paranoico no cree.

La utilización del término creencia me parece acentuada en un sentido menos psicológico de lo que parecería de entrada. La actitud radical del paranoico, tal como Freud la designa, involucra el modo más profundo de la relación del hombre con la realidad, a saber, lo que se articula como la fe. Pueden ver aqui fácilmente cómo se establece el vinculo con otra perspectiva, que llega a su encuentroya se las designé diciéndoles que el mecanismo de la paranoia es esencialmente rechazo de cierto apoyo en el orden simbólico, de ese apoyo específico alrededor del cual puede hacerse -lo veremos en las reuniones siguientes- la división en dos vertientes de la relación con das Ding.

Das Ding es originalmente lo que llamaremos el fuera - de significado. En función de ese fuera - de - significado y de una relación patética con él, el sujeto conserva su distancia y se constituye en un modo de relación, de afecto primario, anterior a toda represión (...).

Pues bien, aqui en relación a ese das Ding original se realiza la primera orientación, la primera elección, el primer emplazamiento de la posición de la orientación subjetiva, que llamaremos en este caso 
Neurosenwahl, la elección de la neurosis. Esta primera muda regulará desde entonces toda la función del principio del placer.

Nos queda aún por ver que en el mismo lugar se organiza algo que es a la vez lo opuesto, lo inverso y lo idéntico y que, en último término, se sustituye a esa realidad muda que es das Ding - a saber la realidad que comanda, que ordena." (Lacan, 2003: 70).

Los últimos parágrafos de esta extensa cita habilitan a extraer las siguientes conclusiones:

a) Cuando hablamos de "posición del sujeto" no estamos haciendo una afirmación en el ámbito de la psicopatología, sino en el de la ética;

b) La "ética" a la cual refiere Lacan no parece estar emparentada simplemente con un "modo de acción" ante tal o cual circunstancia, como podría pensarse partiendo de una lectura ingenua de la "phrónesis" aristotélica, sino que se trata de la posición subjetiva ante el "fuera - de - significado", ante lo Real, ante el "objeto a". En este seminario, ante das Ding, interpretado como "realidad muda".

c) Es exactamente en relación a ese das Ding que se realiza "la primera orientación, la primera elección, el primer emplazamiento de la posición de la orientación subjetiva", y en este sentido, la posición subjetiva del paranoico es utilizada, cambiando de nivel de análisis (del nivel clínico al nivel epistemológico), como un argumento más tendiente a fundamentar la noción lacaniana del "objeto a".

Cabe preguntarse si acaso el discurso del psicoanálisis puede efectivamente pensar por separado la metodología, la ética y la epistemología. Los desarrollos de este breve trabajo harian suponer que no, siendo el seminario VII de Lacan una muestra de ello. Si bien el acento y la 
perspectiva principal de este ensayo ha sido la explicitación epistemológica, cabe señalar que las consecuencias clínicas y metodológicas de este recorrido son iguales o más importantes aún. Pero desarrollarlas claramente excedería los propósitos de este estudio.

Por último, si hay efectivamente una ética analitica, ¿acaso no se trata de distintos posicionamientos subjetivos ante das Ding, es decir ante lo Real?

\section{Referencias bibliográficas}

Allison, H. (1992). El idealismo trascendental de Kant: una interpretación y defensa. Barcelona: Anthropos.

Aristóteles (2004). Ética nicomaquea. Buenos Aires: Losada.

Derrida, J. (1989). "Fuerza y significación". La escritura y la diferencia. Barcelona: Anthropos.

Freud, S. (2000a). Introducción del narcisismo (1914). Obras Completas. Tomo XIV. Bs. As.: Amorrortu.

Freud, S. (2000b). La negación (1925). Obras Completas. Tomo XIX. Bs. As.: Amorrortu.

Freud, S. (2001a). Carta 52 (6/12/1896). Obras Completas. Tomo I. Bs. As.: Amorrortu.

Freud, S. (2001b). Proyecto de psicología (1950 [1895]). Obras Completas. Tomo I. Bs. As.: Amorrortu.

Freud, S. (2001c). Más allá del principio de placer (1920). Obras Completas. Tomo XVIII. Bs. As.: Amorrortu.

Heidegger, M. (1975). La pregunta por la cosa. La doctrina kantiana de los principios trascendentales (1935/36). Buenos Aires: Alfa.

Heidegger, M. (2001). "La cosa” (1951). Conferencias y artículos. Barcelona: Del Serbal. 
Heidegger, M. (2009). El ser y el tiempo (1927). Bs. As.: Fondo de Cultura Económica.

Kant, I. (2003). Crítica de la razón práctica (1788). Buenos Aires: Losada.

Kant, I. (2004). Crítica de la razón pura (1781). Buenos Aires: Losada.

Lacan, J. (1987). "De una cuestión preliminar a todo tratamiento posible de la psicosis" (1957-58). Escritos 2. Bs. As.: Siglo XXI.

Lacan, J. (2001). El seminario. Libro V. Las formaciones del inconsciente (1957-1958). Buenos Aires: Paidós.

Lacan, J. (2002). El seminario. Libro III. Las psicosis (1955-56). Buenos Aires: Paidós.

Lacan, J. (2003). El seminario. Libro VII. La ética del psicoanálisis (19591960). Buenos Aires: Paidós.

Lacan, J. (2006). El seminario. Libro X. La angustia (1962-1963). Buenos Aires: Paidós.

Laplanche, J. (1975). Hölderlin y el problema del padre. Buenos Aires: Corregidor.

Ricoeur, P. (2009). Freud: una interpretación de la cultura (1965). México: Siglo XXI.

Vassallo, S. (2009). El impasse conceptual de la causa en Lacan y las antinomias kantianas. Buenos Aires: El Sigma.com. 